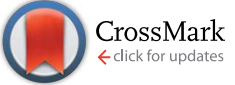

Cite this: RSC Adv., 2017, 7, 1251

\title{
Simultaneous determination of aminoglycoside antibiotics in feeds using high performance liquid chromatography with evaporative light scattering detection
}

\author{
Qingying Liu, $\dagger^{\mathrm{a}}$ Jiufeng $\mathrm{Li}, \dagger^{\mathrm{b}}$ Xuqin Song, ${ }^{\mathrm{a}}$ Meiyu Zhang, ${ }^{\mathrm{a}}$ Erfen Li, ${ }^{\mathrm{b}}$ Fuming Gao ${ }^{\mathrm{b}}$ \\ and Limin $\mathrm{He}^{\star a b}$
}

A sensitive, rapid and reproducible method has been developed based on high performance liquid chromatography with evaporative light scattering detection (HPLC-ELSD) for the simultaneous detection of ten aminoglycoside antibiotics including apramycin, neomycin, amikacin, and gentamicin. The influence of parameters of ELSD on the responses of aminoglycosides was investigated in detail. The target analytes were separated well on a Hypersil BDS $\mathrm{C}_{18}$ column based on ion-pair chromatography. The proposed method was applied to the determination of eight drugs in various animal feeds. Calibration curves of eight aminoglycosides were linear ( $r \geq 0.997)$ within the range of 2.00-200 $\mu \mathrm{g}$ $\mathrm{mL}^{-1}$. The recoveries of eight aminoglycosides from 5 types of animal feeds ranged from $61.2 \%$ to $104.0 \%$ and the intra- and inter-day relative standard deviations were less than $15 \%$. The limits of detection of the eight aminoglycoside drugs were between 0.2 and $0.7 \mathrm{mg} \mathrm{kg}^{-1}$.

Received 10th November 2016
Accepted 7th December 2016

DOI: $10.1039 / c 6 r a 26581 b$

www.rsc.org/advances

\section{Introduction}

Aminoglycoside (AG) antibiotics are a class of compounds whose molecular structure consists of two or more aminosugars participating in a glycosidic linkage to an aminocyclitol component. ${ }^{1}$ The characteristics of AG antibiotics iznclude a broad antibacterial spectrum for some Gram-positive and Gram-negative bacteria. ${ }^{2,3}$ Therefore, numerous farmers tend to employ AGs for the treatment of some parasites and bacterial infections in animals. In recent years, adding antibiotics to various feeds has become more and more popular because the antibiotics can improve the conversion rate of the feed and promote bulk absorption of nutrients for animals. The AGs that have a broad antibacterial spectrum are favored by animal husbandry and veterinary medicine as growth promoters and feed additives. ${ }^{4,5}$ However, the long-term use of the AGs will cause side effects, resulting in potential harm to human health. Typically, AG drug residues in edible animal tissues enter the human body through the food chain, and might cause severe ototoxicity and nephrotoxicity. ${ }^{6}$ Although several countries have banned antibiotics as feed additives, some aminoglycoside

${ }^{a}$ National Reference Laboratory of Veterinary Drug Residues (SCAU), College of Veterinary Medicine, South China Agricultural University, Guangzhou, Guangdong, PC 510642, China. E-mail: liminokhe@scau.edu.cn; Fax: +8620 85284896; Tel: +86 2085280665

${ }^{b}$ Guangdong Provincial Key Laboratory of Veterinary Pharmaceutics Development and Safety Evaluation, China

$\dagger$ The authors contributed equally to this work. antibiotics are still permitted as feed drug additives in China. For example, the dosages of apramycin sulfate and spectinomycin sulfate used in pig's feed are $80-100 \mathrm{mg} \mathrm{kg}^{-1}$ and $22 \mathrm{mg}$ $\mathrm{kg}^{-1}$, respectively. The dosage of neomycin sulfate used in pig's feed is $77 \mathrm{mg} \mathrm{kg}^{-1}$ and in the fowl's feed it is $154 \mathrm{mg} \mathrm{kg}^{-1}$. Consequently, it is indeed necessary to develop convenient, effective and robust analytical methods for the detection and monitoring of AG antibiotics in all types of feeds for animal food safety.

The enzyme linked immunosorbent assays (ELISA) and radioimmunoassay have been commonly used for the determination of aminoglycosides in kidney, milk and some biological samples. ${ }^{8-10}$ However, the aforementioned methods could not provide satisfactory results for the multi-residue quantitation of aminoglycosides. Chromatography is an efficient separation method. Modern high performance liquid chromatography (HPLC) with ultraviolet (UV) and/or fluorescence (FL) detectors has been used to analyze drug residues in animal-derived food. ${ }^{11-13}$ However, because the AGs and their related compounds are without chromophores and fluorophores in their molecular structures, they were usually subjected to pre- or post-column derivatization prior to LC analysis. ${ }^{14,15}$ The derivatives are not always stable and harsh reaction conditions are demanded. In fact, the strategy of liquid chromatographic detection of AGs based on derivatization is not an ideal choice. Liquid chromatography-mass spectrometry (LC-MS) and liquid chromatography tandem mass spectrometry (LC-MS/MS) analysis appear to be widespread methods for 
drugs in complex matrices owing to their high sensitivity and selectivity. ${ }^{16-21}$ However, the determination of AGs in feed using LC-MS and LC-MS/MS has rarely been reported. Furthermore, an evaporative light scattering detector (ELSD) is a sensitive and universal detector used in conjunction with HPLC and is commonly used for the analysis of compounds such as sugars, lipids and novel antibiotics (no UV absorbance) that cannot be detected by a UV detector. Compared with an MS detector, although the ELSD is lower in sensitivity, it is a convenient and low-priced detector, and its sensitivity is enough for the detection of AGs in feeds. There have been some reports on the determination of AGs via HPLC-ELSD. ${ }^{22-24}$ However, these reports still focused on the analysis of single AG compounds and did not apply it to the analysis of real samples. Therefore, it is very important to use HPLC-ELSD for the simultaneous detection of multi-aminoglycoside drugs in animal feeds.

In this study, we focused on the development of a simple, efficient and reliable method for the simultaneous determination of ten aminoglycoside drugs (Fig. 1) using high performance liquid chromatography coupled to an evaporative light scattering detector. Finally, the proposed method was successfully applied to extract and determine eight aminoglycosides in different types of feed samples.

\section{Experimental}

\subsection{Materials and reagents}

The standards of kanamycin (KAN) and apramycin (APR) were obtained from the China Institute of Veterinary Drugs Control (Beijing, China). Spectinomycin (SPC), hygromycin B (HYG), streptomycin (STR), amikacin (AMK), ribostamycin (RIB), tobramycin (TOB), gentamicin (GEN) and neomycin (NEO) were purchased from Dr Ehrenstorfer GmbH Co. (Augsburg, Germany). All of the proposed chemicals were analytical grade with $\geq 87 \%$ purity.

Heptafluorobutyric acid (HFBA) was supplied by Shanghai ANPEL Scientific Instrument Co, Ltd. (Shanghai, China). Acetonitrile (ACN) and methanol were of HPLC grade and purchased from Fisher Scientific (Fair Lawn, NJ, USA). Methanol, glacial acetic acid, concentrated hydrochloric acid $(\mathrm{HCl})$, trichloroacetic acid (TCA), ammonia $\left(\mathrm{NH}_{3} \cdot \mathrm{H}_{2} \mathrm{O}\right)$, ethylenediaminetetraacetic acid (EDTA) disodium salt dihydrate, and monopotassium phosphate $\left(\mathrm{KH}_{2} \mathrm{PO}_{4}\right)$ of reagent grade were supplied by the Guangzhou Chemical Reagent Company (Guangzhou, China). Deionized water was generated using a Millipore system from Millipore (Molsheim France, HPLC grade).

The extraction solvent was $10 \mathrm{mmol} \mathrm{L}{ }^{-1} \mathrm{KH}_{2} \mathrm{PO}_{4}$ containing $0.4 \mathrm{mmol} \mathrm{L}^{-1}$ EDTA and 2\% TCA.

The Bond Elut- $\mathrm{C}_{18}$ solid-phase extraction (SPE) cartridge (500 mg, $5 \mathrm{~mL}$ ) was provided by Agilent Technologies Co. (Santa Clara, CA, USA). The Oasis MCX SPE cartridge (60 mg, $3 \mathrm{~mL}$ ) and Oasis HLB SPE cartridge ( $60 \mathrm{mg}, 3 \mathrm{~mL}$ ) were both bought from Waters Co. (Milford, MA, USA).

Five types of typical animal feeds (A, B, C, D and E) collected from local feed markets were ground and sieved ahead of time (A and B: pig compound feed; C: poultry compound feed; D: pig premix; E: feed additive).

\subsection{Preparation of standard solutions}

To prepare the individual stock standard solutions $\left(5 \mathrm{mg} \mathrm{mL}{ }^{-1}\right)$, $50 \mathrm{mg}$ of each standard was accurately weighed into a $15 \mathrm{~mL}$ polyethylene centrifuge tube. Each compound was dissolved with $10 \mathrm{~mL}$ of water. These solutions were stored at $-20^{\circ} \mathrm{C}$ and stable for up to 1 month. A working solution of ten aminoglycosides (500 $\mu \mathrm{g} \mathrm{mL}^{-1}$ ) was prepared by mixing the stock standard solutions and then diluting stepwise with water to prepare a series of concentrations $\left(50,5.0\right.$ and $\left.0.5 \mu \mathrm{g} \mathrm{mL}{ }^{-1}\right)$. These working solutions were stable for at least two weeks at $4{ }^{\circ} \mathrm{C}$.

\subsection{Instrumentation and chromatographic conditions}

Analysis was carried out on a Waters 2695 Alliance HPLC (Waters Corp., Milford, MA, USA) system consisting of an online vacuum degasser, a quaternary pump solvent management system and an autosampler. The detector was a Waters 2424 ELSD equipped with a GA-10B air generator (Zhongxinghuili Technology Development Co. Ltd., Beijing, China). Empower Software was used for the recording and processing of the chromatographic data.

LC separation was performed on a Hypersil BDS $\mathrm{C}_{18}(250 \mathrm{~mm}$ $\times 4.6 \mathrm{~mm}$ i.d., $5 \mu \mathrm{m}$, Dalian Elite Analytical Instruments Co., Ltd., Dalian, China) reversed-phase chromatography column. The mobile phase A was composed of acetonitrile/water (5 : 95, $\mathrm{v} / \mathrm{v}$ ) and mobile phase B was composed of acetonitrile/water (50:50, v/v), both containing $20 \mathrm{mmol} \mathrm{L}^{-1}$ HFBA. Chromatographic separation of the AG analytes was conducted at room temperature and the elution was carried out at a flow rate of 1 $\mathrm{mL} \min ^{-1}$ with gradient elution mode (Table 1 ). The injection volume was $40 \mu \mathrm{L}$.

The parameters of the ELSD detector were optimized in order to obtain the optimum conditions. The ELSD conditions were as follows: nebulizer gas pressure of 20 psi, drift-tube temperature of $60{ }^{\circ} \mathrm{C}$; gain of 50 and nebulizer temperature of $36{ }^{\circ} \mathrm{C}$. Moreover, the frequency of data collection was set at 1 and the time constant was set at slow speed.

\subsection{Extraction}

One gram of feed sample was accurately weighed into a $15 \mathrm{~mL}$ polypropylene centrifuge tube. First, aliquots $(5 \mathrm{~mL})$ of the extraction solution were transferred into the tube. Furthermore, the tube was vortexed for $1 \mathrm{~min}$ and ultrasonicated for $10 \mathrm{~min}$. Subsequently, the mixture was centrifuged at $10000 \mathrm{rpm}$ for $10 \mathrm{~min}$ at $4{ }^{\circ} \mathrm{C}$. The residues were re-extracted once in the same way. The supernatant was combined into a new centrifuge tube. Before the clean-up procedure, the $\mathrm{pH}$ of the supernatant was adjusted to $5.5 \pm 0.5$ using ammonia.

\subsection{Cleanup}

The MCX SPE cartridge was applied for further sample purification. The cartridge was previously conditioned with $3 \mathrm{~mL}$ of methanol and $3 \mathrm{~mL}$ of $2 \%$ acetic acid. Then, the extracts were carefully transferred onto the cartridge. The flow rate should be slow to avoid weak retention when samples are loaded onto the cartridges, thus a $1 \mathrm{~mL} \mathrm{~min}^{-1}$ flow rate was roughly set. The SPE 
<smiles>NCC[C@H](O)C(=O)N[C@H]1C[C@H](N)[C@@H](O[C@@H]2O[C@H](CN)[C@@H](O)[C@H](O)[C@H]2O)[C@H](O)[C@H]1O[C@@H]1O[C@H](CO)[C@@H](O)[C@H](N)[C@H]1O</smiles>

Amikacin

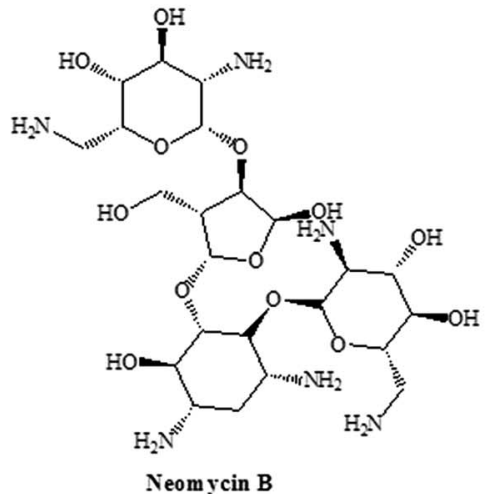<smiles>NC[C@H]1O[C@H](O[C@@H]2[C@@H](N)C[C@@H](N)[C@H](O[C@@H]3O[C@H](CO)[C@@H](O)[C@H](N)[C@H]3O)[C@H]2O)[C@H](N)C[C@H]1O</smiles>

Tobramycin<smiles>CN[C@H]1[C@H](O[C@@H]2O[C@H](CO)[C@@H](N)[C@H](O)[C@H]2O)O[C@@H]2C[C@H](N)[C@@H](O[C@@H]3[C@@H](N)C[C@@H](N)[C@H](O)[C@H]3O)OC2[C@H]1O</smiles>

Apramycin<smiles>NC[C@H]1O[C@@H](O[C@@H]2[C@@H](N)C[C@@H](N)[C@H](O)[C@H]2O[C@@H]2O[C@H](CO)[C@@H](O)[C@H]2O)[C@H](N)[C@H](O)[C@H]1O</smiles>

Ribostamycin

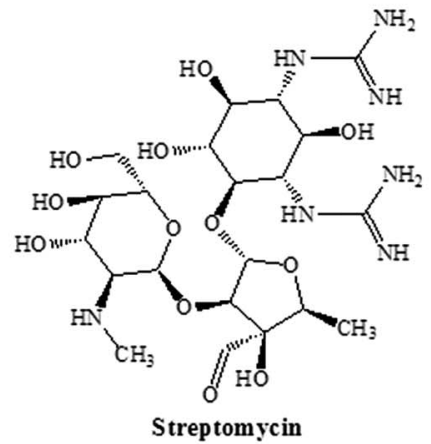<smiles>CN[C@H]1C[C@@H](N)[C@@H](O)[C@H](O[C@@H]2O[C@H](CO)[C@@H](O)[C@H]3OC4(O[C@H]23)O[C@H]([C@H](N)CO)[C@@H](O)[C@H](O)[C@H]4O)[C@@H]1O</smiles>

Hygromycin B<smiles>CN[C@H]1[C@@H](O)[C@@H](NC)[C@H]2C[C@]3(O)C(=O)C[C@@H](C)O[C@@H]3O[C@H]2[C@@H]1O</smiles>

Spectinomycin

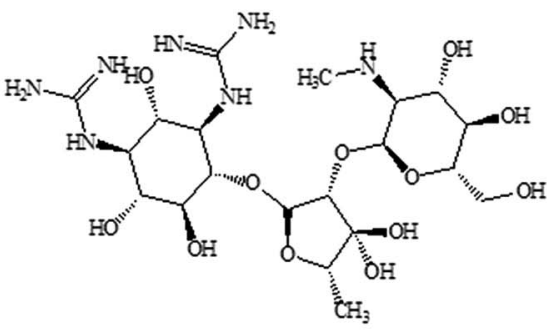

Kanamycin B

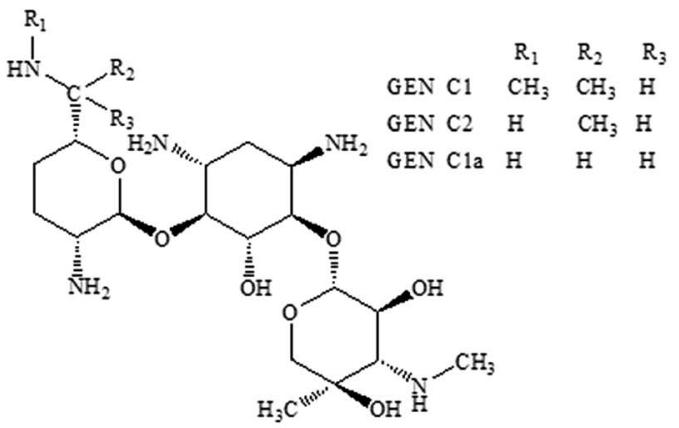

Gentamicin

Fig. 1 Chemical structures of ten aminoglycoside compounds.

cartridges were then washed with $3 \mathrm{~mL}$ of $2 \%$ acetic acid and 3 $\mathrm{mL}$ of water. It was noteworthy that the cartridge should be dried thoroughly. Finally, the target analytes were eluted with 5
$\mathrm{mL}$ of $20 \%$ ammonia in methanol. The eluted solution was collected into a $10 \mathrm{~mL}$ glass tube, followed by evaporating to dryness at $45{ }^{\circ} \mathrm{C}$ under a nitrogen stream. A $1 \mathrm{~mL}$ aliquot of 
Table 1 The HPLC gradient elution program

\begin{tabular}{llll}
\hline Time (min) & A (\%) & B (\%) & Curve $^{a}$ \\
\hline 0 & 65 & 35 & 6 \\
22 & 65 & 35 & 8 \\
23 & 30 & 70 & 6 \\
26 & 30 & 70 & 6 \\
27 & 65 & 35 & 6 \\
35 & 65 & 35 & 6
\end{tabular}

${ }^{a}$ Gradient curve, 6 shows gradient rate unchanged and 8 shows the rate gradually increased.

$20 \mathrm{mmol} \mathrm{L}^{-1}$ HFBA solution was employed in reconstituting the residues. Afterwards, the redissolved solutions were vortexed for $1 \mathrm{~min}$ and filtered through a $0.22 \mu \mathrm{m}$ membrane filter before being injected onto the HPLC-ELSD system.

For the $\mathrm{C}_{18}$ cartridge, the SPE procedure was performed by preconditioning the cartridge with $3 \mathrm{~mL}$ of methanol and $3 \mathrm{~mL}$ of $20 \mathrm{mmol} \mathrm{L}^{-1}$ HFBA. Then, the cartridge was washed with $3 \mathrm{~mL}$ of $20 \mathrm{mmol} \mathrm{L}^{-1} \mathrm{HFBA}$ and $3 \mathrm{~mL}$ of water after loading the sample. Finally, the analytes were eluted with $5 \mathrm{~mL}$ of acetonitrile-100 mmol L $\mathrm{m}^{-1}$ HFBA $(8: 2, \mathrm{v} / \mathrm{v})$. The eluate was blown to dryness and redissolved in $20 \mathrm{mmol} \mathrm{L}^{-1}$ HFBA solution prior to analysis by HPLC.

For the Oasis HLB cartridge, the cartridge was conditioned with $3 \mathrm{~mL}$ of methanol and $3 \mathrm{~mL}$ of water. Sequentially, the cartridge was washed with $3 \mathrm{~mL}$ of water after loading the sample. After drying, the analytes were eluted with $5 \mathrm{~mL}$ of $20 \%$ ammonia in methanol $(\mathrm{v} / \mathrm{v})$. The eluate was blown to dryness and redissolved in $20 \mathrm{mmol} \mathrm{L}^{-1}$ HFBA solution prior to analysis via HPLC.

\section{Results and discussion}

\subsection{Optimization of HPLC conditions}

Aminoglycosides are a class of polar compounds that contain several amino and hydroxyl groups in their molecular structures. These polar groups in AGs have posed to be a major challenge for their retention on traditional chromatographic stationary phases. Hydrophilic interaction liquid chromatography (HILIC) has been a good alternative to realize the chromatographic retention of extremely polar compounds. There are several literature studies that have reported on the determination of AGs using HILIC combined with tandem-mass spectrometry. ${ }^{18,25}$ Practically, the separation selectivity and stability usually cannot entirely meet the determination of most analytes, and sometimes a good peak shape is obtained only through the introduction of a highly concentrated buffer solution, which likely shortens the lifetime of the column; moreover, it is incompatible with MS. However, for strongly polar basic AGs, it is still a common practice to use ion pairing for better selectivity and stability. Generally, the Hypersil BDS $\mathrm{C}_{18}$ column, which has been applied extensively, can acquire good separation and retention for AGs by means of the ion pair reagent. Moreover, this type of column is cheaper than others. Thus, a Hypersil BDS $\mathrm{C}_{18}$ column was selected as the analytical column.
For the mobile phase, acetonitrile and water were the main solvents. ${ }^{20}$ Moreover, HFBA, an acidic ion-pair reagent, played an important role in the mobile phase. It formed an ion-pair complex with AGs, which was retained well in the reversedphase chromatographic column. In our study, concentrations of 5, 10, 15 and $20 \mathrm{mmol} \mathrm{L}^{-1}$ HFBA were tested as the ion-pair reagent. As a result, when $20 \mathrm{mmol} \mathrm{L}^{-1}$ HFBA was used in the mobile phase, all of the target analytes showed high responses and good peak shapes. When the concentration of HFBA in the mobile phase was lower than $20 \mathrm{mmol} \mathrm{L}^{-1}$, there was no evident peak for GEN and NEO. It also demonstrated that higher concentration of HFBA was beneficial to all target AG compounds. However, for decreasing the HFBA damage to the chromatographic column and corrosion of the instrument, a concentration of $20 \mathrm{mmol} \mathrm{L}^{-1}$ HFBA was finally selected for the mobile phase.

The gradient elution conditions were optimized in order to obtain good separation and response. In the actual experiments, we tried to adjust the initial and final proportion of mobile phase B (acetonitrile/water, $50: 50, \mathrm{v} / \mathrm{v}$ ). When the initial proportion of B was set at $40 \%$, the separation was poor for HYG, STR, KAN and RIB (Fig. 2a). The response of SPC was also low. A higher organic phase in the initial proportion of mobile phase B might affect retention of target drugs in the chromatographic stationary phase. Thus, by decreasing the initial proportion of mobile phase B to $35 \%$, each compound could be distinctly separated. Similarly, if the final proportion of mobile phase B was set at $75 \%$, the KAN and RIB were not separated well (Fig. 2b). This is because the selectivity decreased with increases in the percentage of organic solvent in the mobile phase. It was shown that a $70 \%$ proportion of mobile phase $\mathrm{B}$ could achieve good elution.

The gradient duration and curve were further investigated. When the proportion of mobile phase B changed from $35 \%$ to $70 \%$ within 15 min, the HYG and STR could not be completely separated and the response of GEN was low (Fig. 2c). Until $20 \mathrm{~min}$, the HYG and STR still showed poor separation (Fig. 2d). Lastly, in order to realize the complete separation of ten aminoglycosides, a gradient run for $22 \mathrm{~min}$ was selected. As for the gradient curve, if the mobile phase changed according to curve 7 during the 22 min period of the gradient run, the HYG, STR and KAN, RIB could be barely separated and the responses of some drugs presented a downward trend (Fig. 2e). However, when the curve was increased to 9, meaning an acceleration in the rate of change of the mobile phase, the peaks of APR and TOB were not fully resolved from each other (Fig. 2f). Finally, we found that curve 8 was the most suitable for good separation and response of each target analyte. Therefore, curve 8 was selected as the gradient curve. The typical chromatogram of ten aminoglycosides and reagent blank are shown in Fig. $2 \mathrm{~g}$ and $\mathrm{h}$, respectively.

\subsection{Optimization of ELSD parameters}

ELSD has a unique detection principle that can be simply divided into the following steps. First, the effluent from the chromatographic column was nebulized to form an aerosol 

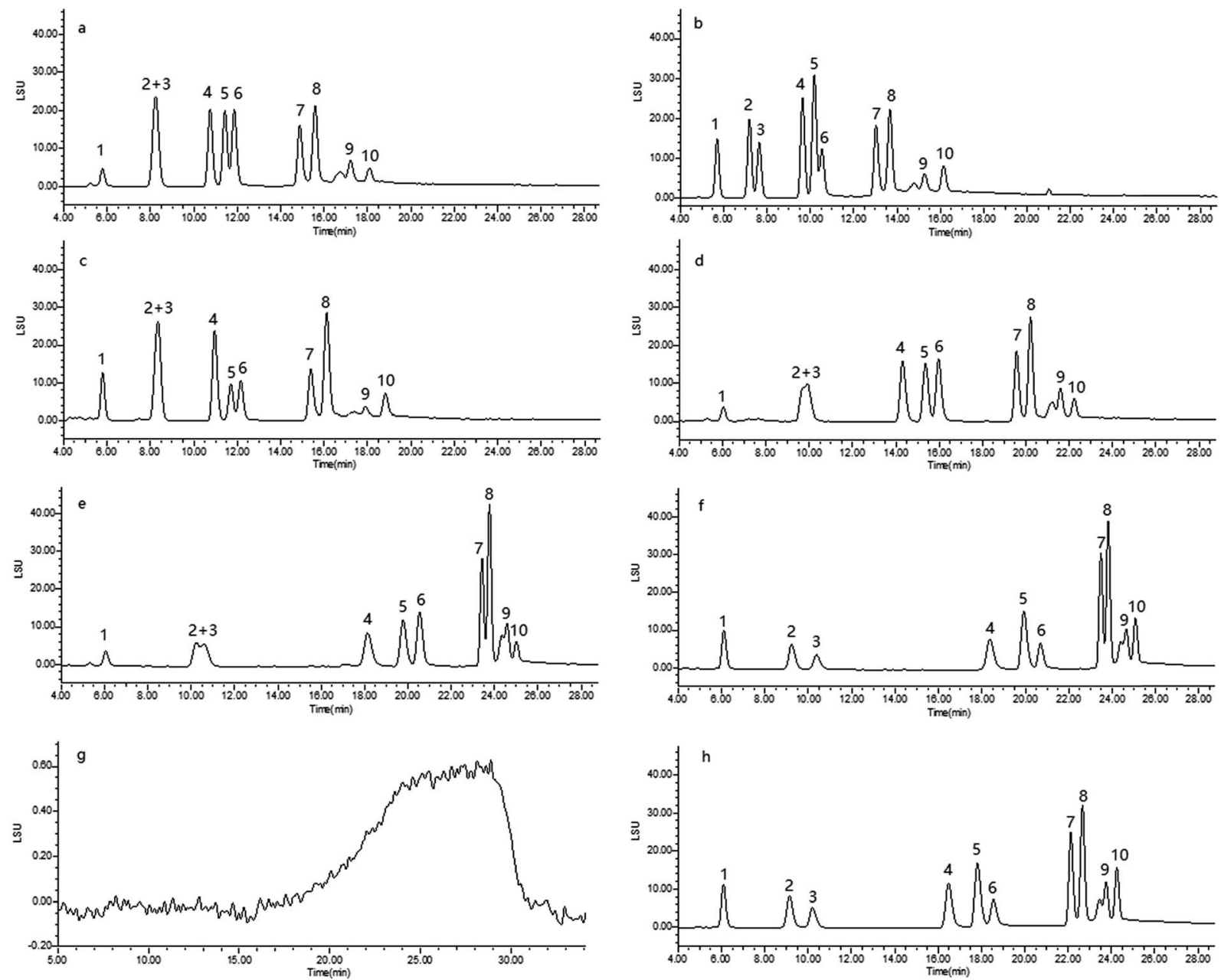

Fig. 2 Influence of different gradient elution conditions on chromatographic separation of ten aminoglycoside compounds (each at $10 \mu \mathrm{g} \mathrm{mL}^{-1}$ ) (a) the initial proportion of B was set at $40 \%$; (b) the final proportion of B was set at $75 \%$; (c) the proportion of B changed from $35 \%$ to $70 \%$ within 15 min; (d) the proportion of B changed from 35\% to 70\% within 20 min; (e) the proportion of B changed from 35\% to 70\% according to curve 7 during $22 \mathrm{~min}$; (f) the proportion of B changed from 35\% to 70\% according to curve 9 during 22 min; ( $g$ ) solvent blank; (h) the optimal separation condition. B, mobile phase B (acetonitrile/water, 50 : 50, v/v). 1, SPC; 2, HYG; 3, STR; 4, AMK; 5, KAN; 6, RIB; 7, APR; 8, TOB; 9, GEN; 10, NEO.

spray and then the solvent was evaporated in a heated drift tube. Second, the remaining non-volatile solute particles were detected in the pool of the light scattering detector. Therefore, the accurate setting of ELSD parameters was very important for the determination of the ten aminoglycosides. In our study, the drift-tube temperature was preferentially optimized. The ten aminoglycosides were analyzed under the drift-tube temperature range from $50{ }^{\circ} \mathrm{C}$ to $100{ }^{\circ} \mathrm{C}$. As shown in Fig. 3, when the temperature increased from $50{ }^{\circ} \mathrm{C}$ to $60{ }^{\circ} \mathrm{C}$, the responses of all compounds were correspondingly enhanced. While the temperature continuously increased to $100{ }^{\circ} \mathrm{C}$, the signal presented a decreasing trend for each compound. It might be that the uniformity of particles or pure solute droplets was destroyed once the drift-tube temperature became too high. Conversely, recommendations were not given to set the drift-tube temperature too low. On one hand, the baseline noise would rise; on the other hand, the solvents that remained would influence the lifetime of the instrument. Consequently, keeping the drift-tube temperature at $60{ }^{\circ} \mathrm{C}$ was extremely ideal for good sensitivity.

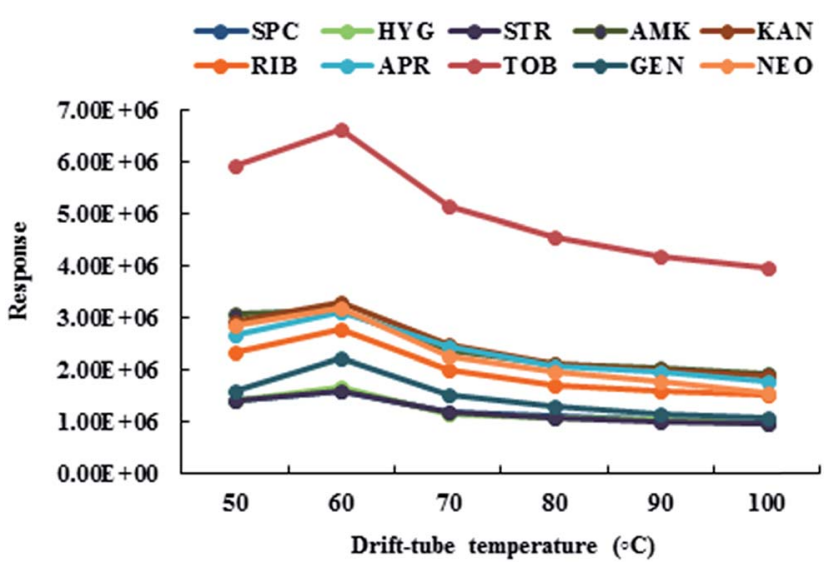

Fig. 3 Influence of drift-tube temperature on responses of ten aminoglycosides. 
The gain of the ELSD was proportional to the baseline noise, and therefore it cannot be too high as it will negatively affect quantitation. In our study, the gain was set at 50 based on responses for all analytes. Furthermore, the data acquisition rate and time constant were set at 1 and slow speed, respectively. The purpose of this was to keep the sensitivity as high as possible.

\subsection{Extraction}

Due to the strong polarity of aminoglycosides, some extraction solvents such as TCA, ${ }^{16,20,26} \mathrm{HCl}^{27}$ and phosphate buffer solution $^{28,29}$ (containing EDTA and TCA) instead of pure organic solvents commonly were used to extract the aminoglycosides from various matrices. In this study, the extraction efficiencies of $10 \mathrm{mmol} \mathrm{L}^{-1} \mathrm{KH}_{2} \mathrm{PO}_{4}$ containing $0.4 \mathrm{mmol} \mathrm{L}^{-1}$ EDTA and $2 \%$ TCA, $2 \%$ TCA, $5 \%$ TCA and $0.1 \mathrm{~mol} \mathrm{~L}^{-1} \mathrm{HCl}$ were investigated by spiking a $50 \mathrm{mg} \mathrm{kg}^{-1}$ level of ten aminoglycosides in feeds. The experimental results showed that the recoveries of all target analytes were very poor (below $40 \%$ ) except for APR, TOB and GEN (above 80\%) when $0.1 \mathrm{~mol} \mathrm{~L}^{-1} \mathrm{HCl}$ was used as an extraction solvent. When the TCA solutions were used, acceptable recoveries could be obtained (above 60\% for each compound). Moreover, weak higher recoveries were gained for most aminoglycosides using 2\% TCA solution than 5\% TCA solution. Fortunately, satisfactory recoveries (above 75\%) were obtained for all the analytes except AMK and NEO (above 66\%) when $10 \mathrm{mmol} \mathrm{L}^{-1} \mathrm{KH}_{2} \mathrm{PO}_{4}$ containing $0.4 \mathrm{mmol} \mathrm{L}^{-1}$ EDTA and $2 \%$ TCA was used as the extraction solvent.

\subsection{Cleanup}

Considering the complex matrix of animal feed, an appropriate purification was required in order to remove the impurities. Bond Elut- $\mathrm{C}_{18},{ }^{30,31}$ Oasis $\mathrm{HLB}^{20,32,33}$ and Oasis $\mathrm{MCX}^{26,34}$ were tested to evaluate the retention of the analytes. For the $\mathrm{C}_{18}$ cartridge, through adding the ion-pair reagent, the retention of analytes was enhanced. As shown in Fig. 4, the majority of analytes obtained low recoveries (less than $65.8 \%$ ), and there was no retention of HYG on the $\mathrm{C}_{18}$ cartridge. For the Oasis HLB cartridge, only APR, TOB, GEN and NEO could be retained. As for the different $\mathrm{p} K_{\mathrm{a}} \mathrm{s}$ of $\mathrm{AG}$ compounds, it failed to achieve the retention of multiple drugs on the HLB cartridge. Moreover, once adjusting the $\mathrm{pH}$ of the extracts to above 6 (a limited pH range), the loading sample solution was immediately turbid. For the Oasis MCX cartridge, it is a mixed-mode sorbent that is capable of retaining basic compounds. Recoveries of all target analytes were above $80 \%$ except for AMK and NEO. It is probably attributed to the AGs being weakly basic compounds. They could easily carry a positive charge due to protonation when the $\mathrm{pH}$ of the extracts was adjusted to 5.5.

Typical chromatograms of blank, spiked samples based on pig compound feed with different SPE cartridges and standard solution of the ten aminoglycosides are shown in Fig. 5. Severe matrix interferences for SPC and STR were observed by cleanup with the $\mathrm{C}_{18}$ SPE cartridge (Fig. 5A). Purification of the extracts by the HLB SPE cartridge also resulted in a slight matrix interference for STR and KAN (Fig. 5B). Clearly, there was also

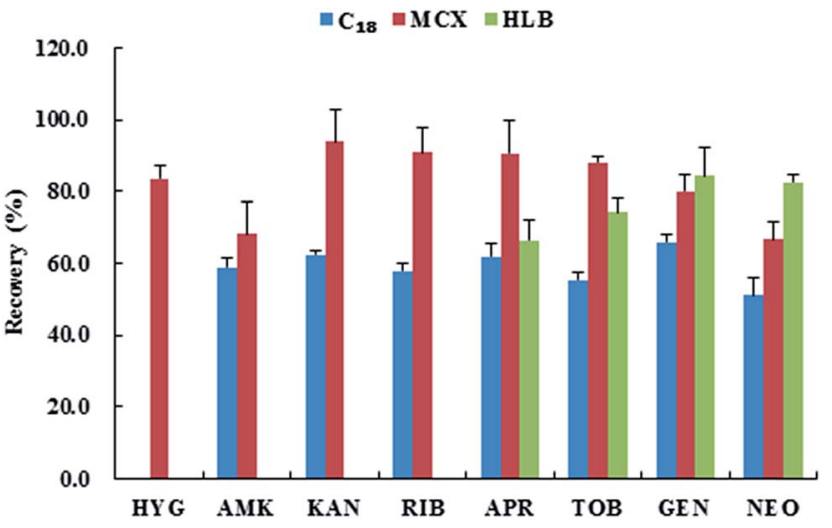

Fig. 4 Recoveries of eight aminoglycosides purified with SPE cartridges (spiking $50 \mathrm{mg} \mathrm{kg}^{-1}$ ).

inevitable matrix interference for SPC and STR even though the extracts were purified by the MCX SPE cartridge. Two severe interfering peaks were observed at the retention times of SPC and STR (Fig. 5C). Their quantification was difficult. Therefore, in order to obtain higher recoveries, better separation and minimize matrix interference to the greatest extent, the MCX cartridge was demonstrated to be the most suitable SPE cartridge for purifying the comparatively complicated animal feed samples.

As for the eluent solution, different concentrations of ammonia in methanol were tested on the MCX SPE cartridge. In general, the recoveries of target analytes gradually improved with an increasing amount of ammonia in methanol. When the eluent was $20 \%$ ammonia in methanol $(\mathrm{v} / \mathrm{v})$, the recoveries of all the analytes were higher than $80 \%$ except AMK and NEO (above $68.5 \%)$. Continually increasing the amount of ammonia in the eluent was not conducive to drying. Thus, $20 \%$ ammonia in methanol was finally selected as the eluent.

\subsection{Performance of the method}

3.5.1. Selectivity. The selectivity was investigated by analyzing 50 blank feed samples. There were interfering peaks at the retention times of SPC and STR in most feed matrices. Therefore, although ten AGs were separated well under optimal chromatographic conditions, only eight of them were quantified in real samples. SPC and STR were neglected due to the lack of a practical strategy to effectively remove endogenous interferences from feed matrices.

3.5.2. Linearity. Calibration curves were established via analyses of spiked blank feed samples of compound feed and feed additive at six concentrations. The results of eight analytes are shown in Table 2. The calibration curves showed good linearity in the range of $2.00-200 \mu \mathrm{g} \mathrm{mL} \mathrm{m}^{-1}$. Correlation coefficients $\left(r^{2}\right)$ were above 0.9970 for eight aminoglycoside drugs.

3.5.3. Recovery and precision. The recovery was determined by spiking eight analytes at three different concentrations of 1.0 (1.5 or 2.0), 10 and $20 \mathrm{mg} \mathrm{kg}^{-1}$ to A, B, C, D and E feed samples over three different days. The recoveries of A, B, C and $\mathrm{D}$ were calculated according to the calibration curve 

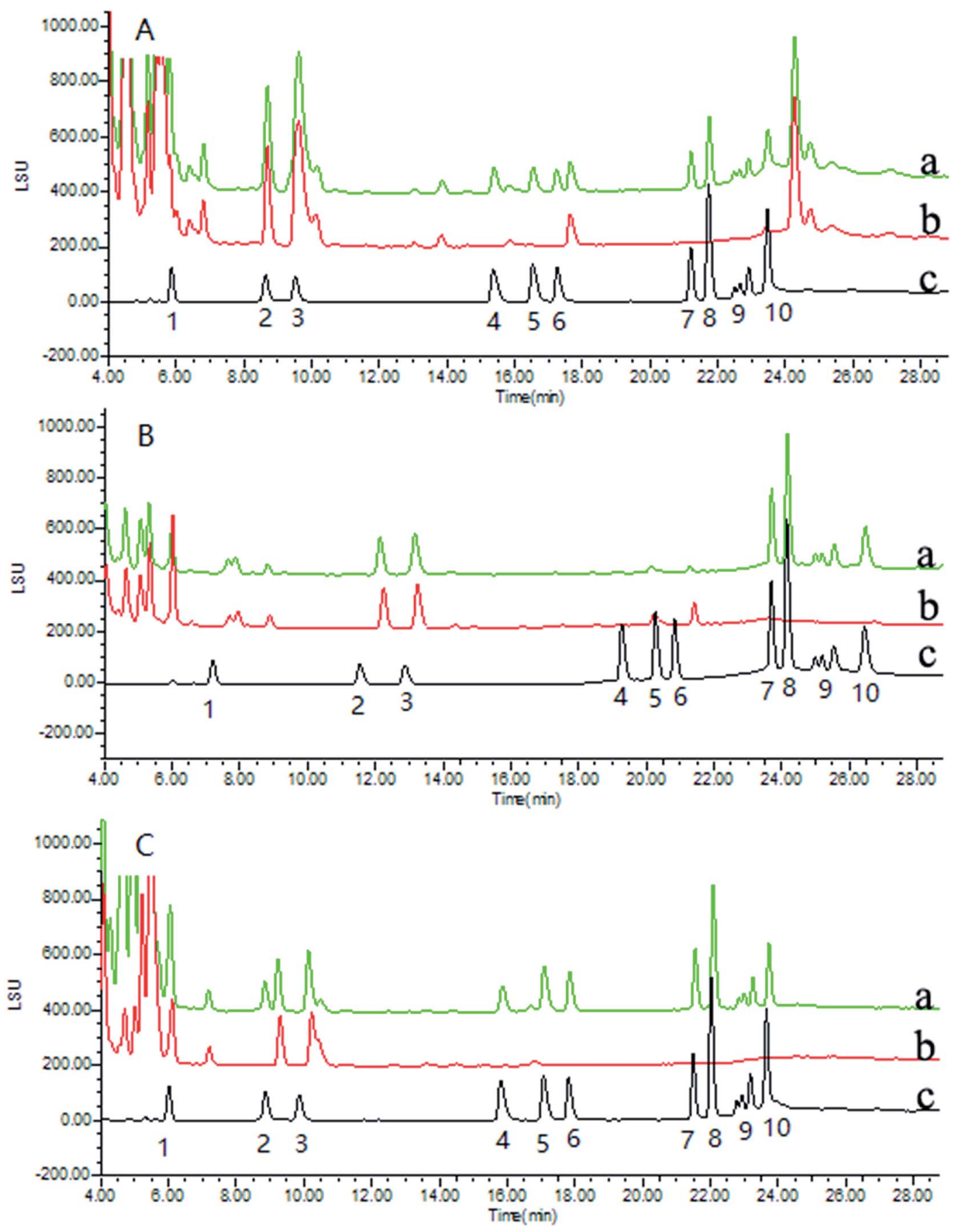

Fig. 5 Typical chromatograms of blank feed spiked at $50 \mathrm{mg} \mathrm{kg}^{-1}$ (a), blank feed (b); standard solution at $50 \mu \mathrm{g} \mathrm{mL}{ }^{-1}$ (c). A, $C_{18}, B, H L B, C, M C X$. Peak identification is the same as in Fig. 2.

of compound feed and $\mathrm{E}$ was calculated according to the calibration curve of feed additive described in Table 2 . The results are shown in Table 3 . The recoveries of eight compounds spiked at three concentrations ranged from $61.2 \%$ to $104.0 \%$. The intra-day relative standard deviations (RSDs) were between $0.4 \%$ and $9.7 \%$ and inter-day RSDs were between $1.1 \%$ and $12.2 \%$.

3.5.4. Limit of detection (LOD) and limit of quantification (LOQ). Two typical feeds (compound feed A and feed additive E) were selected to determine the LOD and LOQ of the proposed method. The values of LOD and LOQ depend on the concentration of eight aminoglycosides giving the signal-to-noise ratio $(\mathrm{S} / \mathrm{N})$ of 3 and 10, respectively. As shown in Table 2, the LOD and LOQ of A and E samples were in the range of $0.2 \mathrm{mg} \mathrm{kg}^{-1}$ (HYG) to $0.7 \mathrm{mg} \mathrm{kg}^{-1}$ (NEO) and $1.0 \mathrm{mg} \mathrm{kg}^{-1}$ (HYG) to $2.0 \mathrm{mg}$ $\mathrm{kg}^{-1}$ (NEO), respectively. This satisfied the requirements to detect the aminoglycosides in real feed samples. 
Table 2 Linearity, LOD and LOQ for eight aminoglycosides

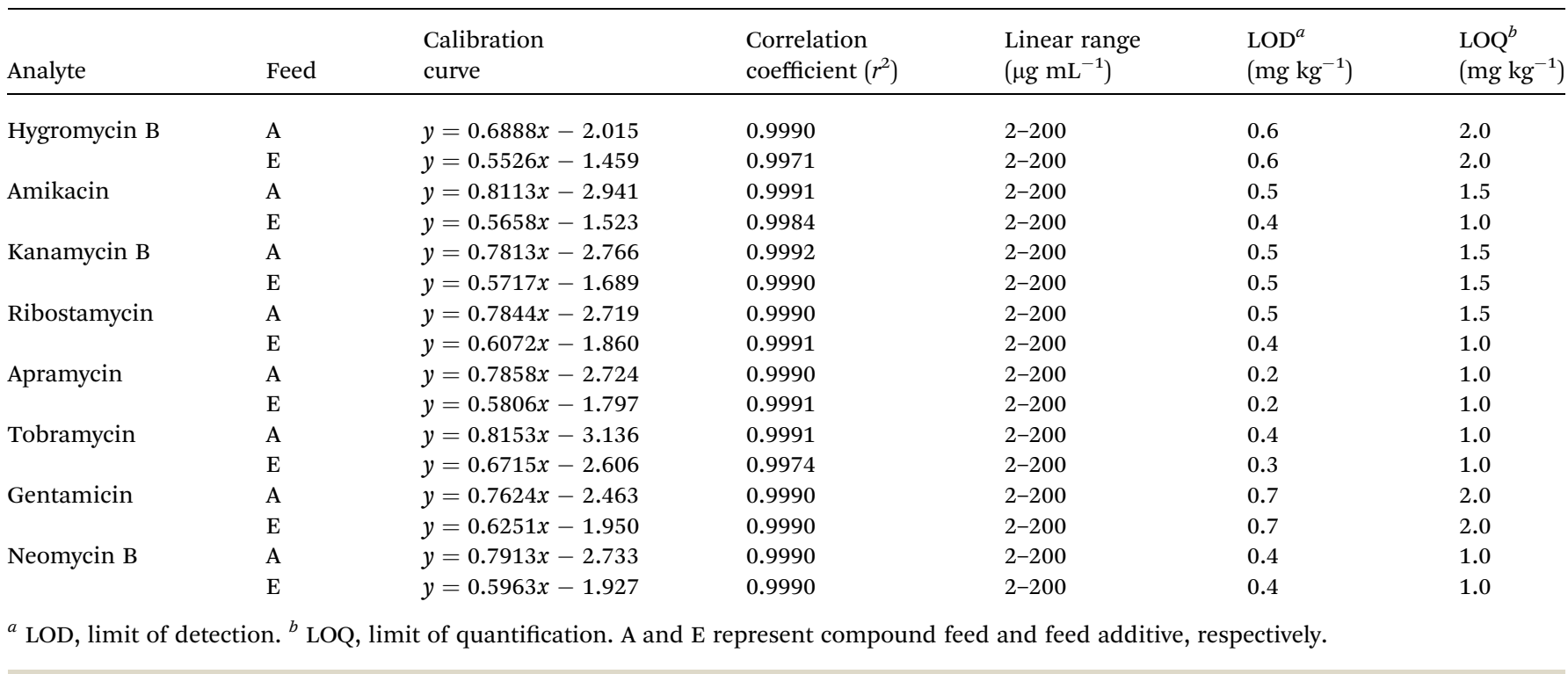

Table 3 Recovery and precision of eight aminoglycosides in five feed samples $(n=3)^{a}$

\begin{tabular}{|c|c|c|c|c|c|c|c|c|c|c|c|}
\hline \multirow{2}{*}{ Analyte } & \multirow{2}{*}{$\begin{array}{l}\text { Spiking } \\
\text { Level } \\
\left(\mathrm{mg} \mathrm{kg}^{-1}\right)\end{array}$} & \multicolumn{10}{|c|}{ verage recovery (RSD), \% } \\
\hline & & \multicolumn{2}{|l|}{ A } & \multicolumn{2}{|l|}{ B } & \multicolumn{2}{|l|}{$\mathrm{C}$} & \multicolumn{2}{|l|}{$\mathrm{D}$} & \multicolumn{2}{|l|}{$\mathrm{E}$} \\
\hline \multirow[t]{2}{*}{ Hygromycin B } & 2.0 & $75.9(7.5)$ & $74.9(10.3)$ & $81.5(3.0)$ & $80.9(7.0)$ & $84.9(8.4)$ & $80.2(8.0)$ & $78.7(1.3)$ & 79.6(1.8) & $91.3(3.6)$ & $92.3(2.9)$ \\
\hline & 10 & $90.6(7.2)$ & $87.9(6.5)$ & $81.0(6.2)$ & $83.7(5.7)$ & $89.0(5.8)$ & $88.1(8.4)$ & $80.8(0.4)$ & $80.6(1.2)$ & $76.1(6.2)$ & $73.1(6.9)$ \\
\hline & 10 & $69.5(9.0)$ & $72.3(7.5)$ & $71.0(0.9)$ & $74.8(7.1)$ & $73.2(2.7)$ & $69.9(6.5)$ & $73.4(0.6)$ & $74.0(2.7)$ & $80.5(2.0)$ & $77.6(3.6)$ \\
\hline & 20 & $81.1(1.8)$ & $78.4(4.6)$ & $69.5(7.5)$ & $67.2(6.2)$ & $73.7(5.4)$ & $77.7(6.5)$ & $78.4(2.0)$ & $73.6(4.3)$ & $69.2(4.9)$ & $74.0(2.9)$ \\
\hline \multirow[t]{3}{*}{ Kanamycin B } & 1.5 & $77.0(3.8)$ & $75.1(3.5)$ & $80.7(5.6)$ & $81.6(4.2)$ & $83.4(3.4)$ & $79.9(10.0)$ & $89.9(6.7)$ & $89.0(5.1)$ & $96.9(0.6)$ & $92.0(6.1)$ \\
\hline & 10 & $79.9(9.0)$ & $79.8(7.5)$ & $72.3(3.1)$ & $74.0(3.2)$ & $77.8(3.1)$ & $84.1(10.2)$ & $84.0(0.8)$ & $82.8(2.3)$ & $79.8(4.7)$ & $76.5(5.0)$ \\
\hline & 20 & $81.1(7.3)$ & $83.5(5.5)$ & $78.9(2.7)$ & $78.6(2.5)$ & $75.3(8.1)$ & $81.9(6.3)$ & $74.1(4.0)$ & $74.7(3.5)$ & $72.0(6.9)$ & $80.7(4.9)$ \\
\hline Ribostamycin & 1.0 & $77.3(9.7)$ & $77.6(11.0)$ & $71.0(2.8)$ & $73.1(10.5)$ & $80.4(4.6)$ & $76.4(7.0)$ & $87.5(0.5)$ & $86.8(2.0)$ & $92.0(4.2)$ & $89.4(6.3)$ \\
\hline & 20 & $78.8(4.9)$ & $76.1(6.2)$ & $88.0(7.3)$ & $85.9(6.1)$ & $91.5(3.3)$ & $90.6(3.5)$ & $97.8(4.1)$ & $95.0(7.8)$ & $75.0(5.5)$ & $78.5(5.2)$ \\
\hline \multirow[t]{3}{*}{ Tobramycin } & 1.0 & $69.9(3.8)$ & $68.7(3.1)$ & $73.7(8.4)$ & $72.4(7.1)$ & $75.9(3.8)$ & $73.7(3.9)$ & $69.4(3.5)$ & $75.9(11.5)$ & $64.3(3.0)$ & $63.6(2.4)$ \\
\hline & 10 & $65.5(1.4)$ & $69.0(5.8)$ & $80.2(4.5)$ & $81.5(3.6)$ & $75.6(0.6)$ & $75.4(1.8)$ & $71.8(6.5)$ & $75.7(11.0)$ & $77.2(6.7)$ & $73.2(7.1)$ \\
\hline & 20 & $75.1(8.0)$ & $79.3(6.9)$ & $85.0(3.5)$ & $85.3(2.6)$ & $67.7(9.3)$ & $70.4(3.3)$ & $73.9(9.6)$ & $70.7(6.6)$ & $64.6(4.4)$ & $61.8(6.0)$ \\
\hline \multirow[t]{3}{*}{ Gentamicin } & 2.0 & $104.0(4.4)$ & $100.5(6.0)$ & $93.1(5.3)$ & $92.0(5.5)$ & $84.6(4.5)$ & $85.7(4.7)$ & $88.8(2.0)$ & $86.8(4.9)$ & $85.7(4.3)$ & $83.7(5.6)$ \\
\hline & 10 & $84.7(4.6)$ & 85.4(10.1) & $98.9(7.0)$ & $99.7(5.3)$ & $84.7(4.2)$ & $87.0(6.6)$ & $93.3(7.0)$ & $93.7(5.6)$ & $81.4(3.9)$ & $79.6(2.9)$ \\
\hline & 20 & $94.2(4.8)$ & $93.6(3.2)$ & $96.7(2.5)$ & $96.6(4.3)$ & $88.4(4.5)$ & $86.1(3.9)$ & $87.3(0.5)$ & $87.7(1.1)$ & $86.1(5.9)$ & $85.0(4.6)$ \\
\hline \multirow[t]{3}{*}{ Neomycin B } & 1.0 & $75.5(4.8)$ & $77.7(4.2)$ & $67.0(7.7)$ & $66.1(5.8)$ & $70.4(0.9)$ & $66.9(4.4)$ & $69.0(4.2)$ & $68.0(5.1)$ & $65.7(5.9)$ & $64.3(5.5)$ \\
\hline & 10 & $66.5(0.7)$ & $68.7(5.8)$ & $64.6(4.3)$ & $65.7(5.3)$ & $72.8(4.7)$ & $68.4(6.2)$ & $77.2(0.4)$ & $72.9(4.8)$ & $80.6(1.7)$ & $76.5(4.9)$ \\
\hline & 20 & $65.1(2.1)$ & $65.7(3.6)$ & $64.8(1.4)$ & $64.5(1.5)$ & $64.8(4.1)$ & $67.5(5.0)$ & $66.7(2.0)$ & $71.6(5.6)$ & $61.2(7.1)$ & $64.2(5.7)$ \\
\hline
\end{tabular}

${ }^{a}$ A and B, pig compound feed; C, poultry compound feed; D, pig premix; E, feed additive. RSD, relative standard deviation.

\section{Conclusion}

A simple and reliable method was successfully developed for the simultaneous determination of ten aminoglycosides via HPLCELSD. Compared with the reported method of aminoglycoside analyses, the proposed method could simultaneously determine multi-aminoglycoside drugs and was further successfully applied to analyze eight aminoglycosides antibiotics in various animal feed samples. The sensitivity of the method is sufficient for the routine monitoring of aminoglycosides in animal feeds. 


\section{Acknowledgements}

The authors are grateful for the financial support from the National Science Foundation of China (31372476 and 31572562), and the Natural Science Foundation of Guangdong Province, China (2015A030313398).

\section{References}

1 D. A. Stead, J. Chromatogr. B: Biomed. Sci. Appl., 2000, 747, 69-93.

2 X. Chu, L. Wu, X. Liu, N. Li and D. Li, Anal. Biochem., 2008, 376, 144-150.

3 K. O. T. Watanabe, K. Matsui and T. Kubota, J. Antimicrob. Chemoth., 1997, 39, 471-476.

4 S. Ji, F. Zhang, X. Luo, B. Yang, G. Jin, J. Yan and X. Liang, J. Chromatogr. A, 2013, 1313, 113-118.

5 T. A. McGlinchey, P. A. Rafter, F. Regan and G. P. McMahon, Anal. Chim. Acta, 2008, 624, 1-15.

6 Y. Cai, Y. e. Cai, J. Cheng, S. Mou and L. Yiqiang, J. Chromatogr. A, 2005, 1085, 124-130.

7 Ministry of Agriculture (MOA), Bulletin, 2001, 168, 2-5.

8 P. S. Willem Haasnoot, G. Cazemier, A. Lommen, J. F. M. Nouws and A. H. J. Keukens, Analyst, 1999, 124, 301-305.

9 F. Diana, M. Paleologo and L. Persic, Food Addit. Contam., 2007, 24, 1345-1352.

10 M. Shalev, J. Kandasamy, N. Skalka, V. Belakhov, R. RosinArbesfeld and T. Baasov, J. pharmaceut. Biomed., 2013, 75, 33-40.

11 A. V. S. E. Kaale, E. Roets and J. Hoogmartens, J. Chromatogr. A, 2001, 924, 451-458.

12 I. Baranowska, P. Markowski and J. Baranowski, Anal. Chim. Acta, 2006, 570, 46-58.

13 Y. F. Lin, Y. C. Wang and S. Y. Chang, J. Chromatogr. A, 2008, 1188, 331-333.

14 C. Z. Yu, Y. Z. He, G. N. Fu, H. Y. Xie and W. E. Gan, J. Chromatogr. B: Anal. Technol. Biomed. Life Sci., 2009, 877, 333-338.

15 P. Vinas, N. Balsalobre and M. Hernandez-Cordoba, Talanta, 2007, 72, 808-812.
16 Y. Tao, D. Chen, H. Yu, L. Huang, Z. Liu, X. Cao, C. Yan, Y. Pan, Z. Liu and Z. Yuan, Food Chem., 2012, 135, 676-683.

17 S. I. Kotretsou, Crit. Rev. Food Sci. Nutr., 2004, 44, 173-184.

18 P. A. Martos, F. Jayasundara, J. Dolbeer, W. Jin, L. Spilsbury, M. Mitchell, C. Varilla and B. Shurmer, J. Agric. Food Chem., 2010, 58, 5932-5944.

19 J. B. Arsand, L. Jank, M. T. Martins, R. B. Hoff, F. Barreto, T. M. Pizzolato and C. Sirtori, Talanta, 2016, 154, 38-45.

20 W. X. Zhu, J. Z. Yang, W. Wei, Y. F. Liu and S. S. Zhang, J. Chromatogr. A, 2008, 1207, 29-37.

21 M. Rodriquez, D. S. Cretoso, M. A. Euterpio, P. Russo, C. Crescenzi and R. P. Aquino, Anal. Bioanal. Chem., 2015, 407, 7691-7701.

22 I. Clarot, P. Chaimbault, F. Hasdenteufel, P. Netter and A. Nicolas, J. Chromatogr. A, 2004, 1031, 281-287.

23 J. Zhou, L. Zhang, Y. Wang and C. Yan, J. Sep. Sci., 2011, 34, 1811-1819.

24 N. C. Megoulas and M. A. Koupparis, Anal. Bioanal. Chem., 2005, 382, 290-296.

25 C. Diez, D. Guillarme, A. Staub Sporri, E. Cognard, D. Ortelli, P. Edder and S. Rudaz, Anal. Chim. Acta, 2015, 882, 127-139.

26 E. Alechaga, E. Moyano and M. T. Galceran, Anal. Bioanal. Chem., 2014, 406, 4941-4953.

27 Y. X. Zhou, W. J. Yang, L. Y. Zhang and Z. Y. Wang, J. Liq. Chromatogr. Relat. Technol., 2007, 30, 1603-1615.

28 F. L. van Holthoon, M. L. Essers, P. J. Mulder, S. L. Stead, M. Caldow, H. M. Ashwin and M. Sharman, Anal. Chim. Acta, 2009, 637, 135-143.

29 M. P. Almeida, C. P. Rezende, L. F. Souza and R. B. Brito, Food Addit. Contam., Part A, 2012, 29, 517-525.

30 D. N. Heller, J. O. Peggins, C. B. Nochetto, M. L. Smith, O. A. Chiesa and K. Moulton, J. Chromatogr. B: Anal. Technol. Biomed. Life Sci., 2005, 821, 22-30.

31 Y. Wang, S. Li, F. Zhang, Y. Lu, B. Yang, F. Zhang and X. Liang, J. Chromatogr. A, 2016, 1437, 8-14.

32 R. H. Granja, A. M. Nino, R. A. Zucchetti, R. E. Nino, R. Patel and A. G. Salerno, Anal. Chim. Acta, 2009, 637, 64-67.

33 Z. Zhu, G. Liu, F. Wang, J. J. Sasanya and A. Cannavan, Food Anal. Methods, 2016, 9, 2587-2599.

34 R. Oertel, V. Neumeister and W. Kirch, J. Chromatogr. A, 2004, 1058, 197-201. 